\title{
Über das Schicksal des infundierten Zuckers mit besonderer Berücksichtigung der Zuckeraufnahmefähigkeit der Gewebezellen.
}

\author{
VI. Mitteilung. \\ Verhalten des Laktazidogens, der Milchsäure und \\ des Inosits im Muskel nach Zuckerzufuhr.
}

Von

Dr. Kaoru Sato, Dr. Kosaku Ueda und Dr. Toshio Kurokawa. (佐 藤 䓵) (上田耕作) (黑 川 利 雄)

(Aus der Mfedizinischen Klinik von Prof. S. Yamakawa an der Universität zu Sendai.)

Einer $^{1)}$ von uns hat schon bei früheren Versuchen öfters gezeigt, dass intravenös einverleibter Traubenzucker grösstenteils schnell von den Gewebezellen aufgenommen und als nicht reduzierende Substanz aufgestapelt wird. Die Verbindung dieses modifizierten Zuckers mit Körpereiweiss ist gar nicht fest, er geht leicht in den wässerigen Auszug über. Der Muskelauszug des mit Zucker belasteten Tiers weist deshalb immer einen deutlichen Mehrbetrag des $\mathrm{C}$ auf, im Verhältnis zu dessen N-Gehalt. Nach diesem Befunde muss man zur Identifizierung des modifizierten Zuckers vor allem an die C-haltigen und $\mathrm{N}$-freien Extraktivstoffe des Muskels denken. Da aber Glykogen und seine Ablömmlinge sich durch Reduktionsvermögen ohne weiteres davon unterscheiden lassen, so kommen danach Laktazidogen, Milchsäure und Inosit in Betracht.

\section{Laktazidogen und Milchsïure.}

Die Zwischenstufe des Kohlehydratstoffwechsels ist bekanntlich neuerdings von Meyerhof ${ }^{2>}$ besonders gründlich bearbeitet worden und verschiedene Substanzen wurden ihr eingereiht. Viele dieser Substanzen sollen

1) Kurokawa, T., Tohoku Journ. of Exp. Med., 1928, 10, 64, 76, 87, 198 und 209.

2) Meyerhof, O., Klin. Wochenschr., 1922, 2. Jg, 230. 
aber sehr unbeständig sein, und deshalb sind sie für unsern Versuch, der nach dem unbekannten C-haltigen Extraktivstoff chemisch forscht, sehr ungeeignet. Unter anderen sind jedoch Laktazidogen und Milchsäure zur quantitativen Untersuchung unschwer zugänglich.

\section{Versuchsmethodik.}

Kaninchen, die 24 Stunden lang vor dem Versuche gehungert hatten, werden auf dem Operationsbrett in Rückenlage fixiert; nachdem man zuerst A. iliaca comm. sin., die die hintere Extremitüt auf derselben Seite versorgt, unterbunden hat, injiziert man $10 \mathrm{~cm} 50$;óiger Traubenzuckerlösung pro kg Körpergewicht langsam in die Ohrvene. 2 Minuten danach werden die Tiere getötet und die gleichnamige Schenkelmuskulatur aus beiden Seiten resp. aus der zirkulierenden und nicht zirkulierenden Seite möglichst schnell herausgeschnitten. von anhaftendem Fett- und Bindegewebe befreit, sofort mit flüsiger Luft gefroren und unter erniedrigter Temperatur im Mörser gebrauchsfertig zerkleinert. Mit dieser pulrerisierten Muskelmasse wird einerseits die Laktazidogenphosphorsäure nach $\mathbf{E}_{\mathbf{m}}$ b d en ${ }^{\mathbf{1}}$ gravimetrisch, andererseits die Milchsïure nach $\mathrm{Su} \mathrm{to}^{2}$ ) durch Extraktionsmethode ermittelt.

\section{Versuchsergebnisse.}

\section{Vergleich der Muskelmasse beider Schenkelseiten bez. des} Gehalts an Laktazidogen und Milchsäure.

\section{1) Leerversuch.}

Wie es in den früheren Mitteilungen immer der Fall war, ist es auch in vorliegender Arbeit notwendig, einen Leerversuch vorauszuschicken, in dem die Muskelmassen der beiden hinteren Extremitäten ohne vorherige Zuckerinfusion auf ihre zu untersuchenden Bestandteile geprüft werden. Ca. 5 Minuten nach Unterbindung von $A$. iliaca comm. sin. wurde das Kaninchen getötet, damit wir den Gehalt an Laktazidiogen und Milchsäure in der gleichnamigen Muskelmasse der beiden hinteren Extremitäten miteinander vergleichen konnten.

Wie ans nebenstehender 'Tabclle ersichtlich, schwanken die beiden Substanzen individuell betrüchtlich; bei Laktazidogen ist die Schwankung zwischen 0,176 und $0,244 \%$ und bei Milchsïure zwischen 0,145 und $0,225 \%$. Dabei besteht aber kein merklicher Unterschied zwischen den beiden Seiten desselben Individuums, denn er beträgt höchstens $0,02 \%$.

1) Embden, G., Z. f. physiol. Chem., 1921, 113, 138.

2) Suto, K., S. T anak a und M. Endo, Juzenkai Zasshi, 1927, 32, 1910. 
Tabelle I.

Laktazidogen- und Milchsiuregehalt der Muskeln der beiden Schenkelseiten bei unbehandelten Kaninchen.

\begin{tabular}{|c|c|c|c|c|}
\hline \multirow{2}{*}{$\begin{array}{l}\text { Kaninchen- } \\
\text { Nr. }\end{array}$} & \multirow{2}{*}{ Körpergewicht } & \multirow{2}{*}{$\begin{array}{l}\text { Seite d. } \\
\text { Schenkels }\end{array}$} & \multicolumn{2}{|c|}{$100 \mathrm{~g}$ frischen Muskels enthalten : } \\
\hline & & & $\begin{array}{c}\text { Laktazidogen } \\
(\mathrm{g})\end{array}$ & $\begin{array}{l}\text { Milchsiure } \\
\qquad(\mathrm{g})\end{array}$ \\
\hline 1 & 2,0 & $\mathbf{L}$ & $\begin{array}{l}0,2042 \\
0.9485\end{array}$ & $\begin{array}{l}0,195 \\
0,005\end{array}$ \\
\hline 2 & 1,8 & $\mathrm{~L}$ & $\begin{array}{l}0,1886 \\
0,1927\end{array}$ & $\begin{array}{l}0,142 \\
0,163\end{array}$ \\
\hline & & $\mathrm{L}$ & 0,1766 & 0,188 \\
\hline 3 & 2,0 & $\mathrm{R}$ & 0,1802 & 0,198 \\
\hline 4 & $\mathbf{2}, 0$ & $\mathrm{~L}$ & 0,2002 & 0,206 \\
\hline 5 & 18 & $\mathrm{~L}$ & 0,2166 & 0,145 \\
\hline 0 & 1,8 & $\mathrm{R}$ & 0,2245 & 0,148 \\
\hline
\end{tabular}

2) Belastung der Tiere mit Zucker.

Die Versuchstiere, denen vorher A. iliaca comm. sin. unterbunden war, erhielten $10 \mathrm{ccm} 50 \%$ iger Traubenzuckerlösung pro kg zweimal hintereinander mit einem Intervalle von einer Minute in die Ohrvene. In der Tabelle II bemerkt man keinen merklichen Unterschied in Milchsäureoder Laktazidogengehalt zwischen der Kontroll- und der mit Zucker belasteten Seite.

Tabelle II.

Laktazidogen- und Milchsiiuregehalt der Muskeln der beiden Schenkelseiten bei nit Zucker behundelten Kaninchen.

\begin{tabular}{|c|c|c|c|c|}
\hline \multirow{2}{*}{$\begin{array}{l}\text { Kaninchen- } \\
\mathrm{Nr} \text {. }\end{array}$} & Körpergewicht & \multirow{2}{*}{$\begin{array}{l}\text { Seite d. } \\
\text { Schenkels }\end{array}$} & \multicolumn{2}{|c|}{$100 \mathrm{~g}$ frischen Muskels enthalten } \\
\hline & $(\mathrm{kg})$ & & $\begin{array}{c}\text { Laktazidogen } \\
\text { (g) }\end{array}$ & $\begin{array}{l}\text { Milchsïure } \\
\text { (g) }\end{array}$ \\
\hline 6 & 1,6 & $\begin{array}{l}\mathrm{L} \\
\mathrm{R}\end{array}$ & $\begin{array}{l}0,1717 \\
0,1539\end{array}$ & $\begin{array}{l}0,168 \\
0,156\end{array}$ \\
\hline 7 & 1,7 & L & $\begin{array}{l}0,2022 \\
0,1804\end{array}$ & $\begin{array}{l}0,183 \\
0,162\end{array}$ \\
\hline 8 & 1,5 & $\mathrm{~L}$ & $\begin{array}{l}0,1562 \\
0,2756\end{array}$ & $\begin{array}{l}0,183 \\
0,198\end{array}$ \\
\hline 9 & 1,6 & $\mathrm{~L}$ & $\begin{array}{l}0,1694 \\
0,2108\end{array}$ & $\begin{array}{l}0,126 \\
0,147\end{array}$ \\
\hline 10 & 2,2 & $\mathrm{~L}$ & 0,2228 & 0,327 \\
\hline & & $\mathrm{R}$ & $\begin{array}{l}0,2508 \\
0,2343\end{array}$ & $\begin{array}{l}0,309 \\
0,227\end{array}$ \\
\hline 11 & 1,9 & $\mathrm{R}$ & 0,2496 & 0,248 \\
\hline 12 & 2,6 & $\begin{array}{l}\mathrm{L} \\
\mathrm{R}\end{array}$ & $\begin{array}{l}0,1590 \\
0,2771\end{array}$ & $\begin{array}{l}0,147 \\
0,227\end{array}$ \\
\hline
\end{tabular}




\section{Inosit.}

Aus Obigen erhellt, dass an dem Mehrbetrag des C im Muskelextrakt, der durch Zuckerzufuhr hervorgerufen wird, Laktazidogen oder Milchsäure nicht nachweisbar beteiligt sind. Es gibt noch einen anderen Nfreien Extraktivstoff im Muskel, nümlich Inosit, der zuerst von Sch ere $\mathbf{r}^{1)}$ entdeckt und von Maquen ne $\mathrm{e}^{2}$ als Hexaoxyhydorbenzol, also als eine mit Glukose isomere Substanz $\left(\mathrm{C}_{6} \mathrm{H}_{12} \mathrm{O}_{6}\right)$ erkannt wurde. Die Beziehung des Inosits zum Zucker wird natürlich dadurch nicht erwiesen, jedenfalls ist jener in seinem chemischen Verhalten mit den Zuckerarten nicht nahe verwandt, da er weder reduziert noch vergärt. Über die Stellung des Inosits im Stoffwechsel sind wir noch nicht genügend unterrichtet. Starkenstein ${ }^{3}$ sieht den freien Inosit nur als wertloses Spaltprodukt an, was aber nicht unwidersprochen geblieben ist. Mayer ${ }^{4)}$ betrachtet den Inosit als einen Milchsäurebildner, da es ihm gelang, den Übergang von Inosit in Gärungsmilchsïure im Körper nachzuweisen. Auch bestätigten andere Autoren (Needham, Smith und Winter) ${ }^{5)}$ Vermehrung des Muskelinosits nach Insulininjektion. Nach diesen Beobachtungen ist es nicht vollständig ausgeschlossen, dass der Inosit noch mit dem Kohlehydratstoffwechsel in einem gewissen Zusammenhang steht. Da wir jetzt zur Erkenntnis des noch unbekannten modifizierten Zuckers, der den Mehrbetrag des C im Muskel verursacht, eine gewisse, nicht reduzierende C-haltige und N-freie Substanz finden müssen, so wird auch Inosit bei der Untersuchung in Betracht gezogen.

Die Versuchsanordnung ist folgende. Als Versuchstiere wïhlten wir mittelgrosse Kaninchen, die vor dem Versuche 24 Stunden lang gehungert hatten. Als Kontrolle diente die Muskelmasse von ohne Vorbehandlung getöteten Tieren. Den Hauptversuchstieren wurden pro $\mathrm{kg} 10 \mathrm{~cm} 50$,áiger Traubenzuckerlösung in Intervallen von 2 Minuten dreimal wiederholt in die Ohrvene gespritzt. Sofort nach der letzten Infusion wurden die Kaninchen bis zum Tode entblutet und die Skelettmuskeln in der zur Untersuchung nötigen Menge möglichst schnell herauggeschnitten. Die Bestimmungsmethode des Inosits ist bisher uberhaupt noch nicht geniigend sicher gestellt worden. Die früberen Angaben beziehen sich zumeist auf die Darstellungsmethode. Bei unserem Versuche, dessen Ziel in der Identifizierung einer unbekannten, nach Zuckerinfusion ins Muskeldekokt übergehenden, C-haltigen Substanz besteht, ist ein wäzseriger Muskelauszug als Ausgangsmaterial im voraus gegeben. Deshalb verwendeten wir zuerst die von $R$ osenberger, ${ }^{(3)}$ Scherer $r^{7)}$ und $\mathrm{M}$ ülle $\mathrm{r}^{8)}$ zur Inositdar-

1) Scherer, Liebigs Annalen, 1850, 73, 322.

2) Maquen ne, Compt. rend. de l'Acad. des Sc., 1887, 104, 297.

3) Starkenstein, E., Biochem. Z., 1910, 30, 56.

4) Mayer, P., Biochem. Z., 1907, 2, 398.

5) Needham, J., Smith, W., und Winter, L. B., J. Physiol., 1923, 82, 57.

6) Rosenberger, F., Z. f. physiol. Chem., 1908, 56, 373.

7) Scherer, Liebigs Annalen, 1850, 73, 322.

8) Müller, Liebigs Annalen, 1857, 103, 140. 
stellung ausgearbeitete Wasserextraktionsmethode, und danach auch die Azetonextraktionsmethode, womit kïrzlich $\mathrm{Needh}$ a $\mathrm{m}^{1}$ den Inositgehalt des Gehirns bestimmt hat.

Bei diesen Untersuchungen haben wir aber auf den Reinigungsversuch verzichtet, weil die Ausbente dadurch sehr verringert werden müsste. Wir begnügten uns hier damit, den C-Gehalt des roh ausgefällten Inositanteils des Muskelextrakts zu bestimmen und den dadurch gewonnenen Schätzungswert des Hauptversuchstiers mit dem des Kontrollversuchstiers zu vergleichen.

1) Fällung mit Bleiazetat.

Die Methode von Scherer, Müller u. a. m. basiert darauf, dass der Inosit nicht von Bleizucker, wohl aber von Bleiessig in Sieden gefällt wird.

$200 \mathrm{~g}$ frische Muskeln, die von Fett und Sehne möglichst frei waren, wurden mit der Hackmaschine zerkleinert, in kochendes Wasser getan und eine Stunde lang im Dampftopf erhitzt. Das Dekokt wurde vorsichtig dekantiert und der Bodensatz wiederum mit destilliertem $W$ asser eine Stunde lang ausgekocht. Dus Dekokt wurde mit dem Spülwasser vereinigt, in einer Abdampfschale auf Wasserbad bis auf ca. $500 \mathrm{ccm}$ eingeengt. Zu diesem rohen Extrakt wurden $10 \mathrm{~g}$ Bleizucker und $5 \mathrm{ccm}$ Eisessig zugefügt, und dies wurde filtriert. Aus dem klaren Filtrat wurde der Inosit durch Zusatz ron Bleiessig und Ammoniak gefiallt, durch Membranfilter filtriert und gut gewaschen. Der Niederschlag wurde im Wasser sus-

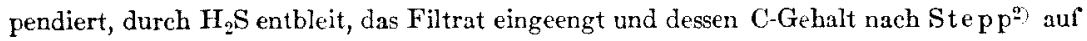
nassem Weg verarbeitet.

Das Ergebnis ist in der Tabelle III zusammengestellt. Man sieht zwischen dem Haupt- und Kontrollversuch keinen merklichen Unterschied des Inositanteils.

\section{Tabelle III.}

Inositgehalt des Skelettmuskels bei Kontroll- und Zuckertieren.

\begin{tabular}{c|c|c}
\hline $\begin{array}{c}\text { Kontrolltier- } \\
\text { Nr. }\end{array}$ & $\begin{array}{c}\text { Körpergewicht } \\
(\mathrm{kg})\end{array}$ & $\begin{array}{c}100 \mathrm{~g} \text { frischen Muskels enthalten : } \\
\text { Inosit } \\
(\mathrm{g})\end{array}$ \\
\hline & 1,8 & 0,005 \\
2 & 2,0 & 0,014 \\
3 & 1,9 & 0,010 \\
Zuckertier-Nr. & & Mittel $=\mathbf{0 , 0 1 1}$ \\
1 & 2,0 & 0,021 \\
2 & 2,0 & 0,014 \\
3 & 1,8 & 0,009 \\
4 & 1,7 & 0,021 \\
& & Mittel $=\mathbf{0 . 0 1 6}$
\end{tabular}

1) Need h am, J., Biochem. Journ., 1923, 17, 422.

2) Stepp, W., Biochem. Z., 1918, 87, 185. 
2) Zuletzt wurde die Bestimmungsmethode von Needham herangezogen, derzufolge der Inosit am besten mit Azeton extrahiert wird.

$200 \mathrm{~g}$ zerkleinerte Muskeln wurden in $200 \mathrm{~cm}$ Azeton krïftig geschüttelt, ca. 18 Stunden lang in Zimmertemperatur stehen gelassen und durch Gaze koliert. Die Behandlung mit Azeton wurde wiederholt, und die vereinigten Extrakte wurden gerade bis zum Sieden erhitzt und durch Papier filtriert. Von dem klaren, gelblich gefürbten Filtrat wurde Azeton anfangs im Vakuum und dann, nach Zusatz von Wasser, auf siedendem Wasserbade griindlich verdunstet. Darauf wurde die Lösung zuerst mit neutralem, dann mit basischem Bleiazetat behandelt. Der Inosit wurde dann wie beim vorigen Versuch durch $\mathrm{H}_{2} \mathrm{~S}$ von der Bleiverbindung getrennt und schliesslich mit Alkohol und Äther als weisslicher Niederschlag gef:̈llt. Der Niederschlag wurde durch Verdunsten von anhaftendem Alkohol und Äther befreit und die C-Bestimmung wie in obigen Versuchen ausgeführt.

Tabelle IV gibt das Ergebnis des Versuchs wieder.

\section{Tabelle IV.}

Inositgehalt des Skelettmuskels bei Kontroll- und Zuckertieren.

\begin{tabular}{c|c|c}
\hline $\begin{array}{c}\text { Kontrolltier- } \\
\text { Nr. }\end{array}$ & $\begin{array}{c}\text { Körpergewicht } \\
(\mathrm{kg})\end{array}$ & $\begin{array}{c}100 \mathrm{~g} \text { frischen Muskels enthalten : } \\
\text { Inosit } \\
(\mathrm{g})\end{array}$ \\
\hline & 1,8 & 0,018 \\
2 & 2,0 & 0,022 \\
3 & 1,8 & 0,023 \\
Zuckertier-Nr. & & Mittel $=\mathbf{0 , 0 2 1}$ \\
1 & 1,8 & 0,022 \\
2 & 1,8 & $\mathbf{0 , 0 2 4}$ \\
3 & 2,1 & $\mathbf{0 , 0 2 6}$ \\
& & Mittel $=\mathbf{0 , 0 2 4}$
\end{tabular}

Man findet hier auch keinen wesentlichen Unterschied des Inositanteils bei Kontroll- und Zuckertieren.

\section{Zusammenfassung.}

Im Muskelextraht mit Traubenzucker behandelter Tiere, der im Vergleich zu dem der Kontrolltiere einen Mehrbetrag an $\mathrm{C}$ aufweist, wird weder Laktazidogen, noch Milchsäure, noch Inosit vermehrt gefunden. 